\title{
Mechanistic and functional diversity in the mechanosensory kinases of the titin-like family
}

\author{
Olga Mayans*1, Guy M. Benian†, Felix Simkovic* and Daniel J. Rigden* \\ "Institute of Integrative Biology, Biosciences Building, University of Liverpool, Crown Street, Liverpool L69 7ZB, U.K., and †Department of Pathology, \\ Whitehead Biomedical Research Building, Emory University, Atlanta, GA 30322, U.S.A.
}

\begin{abstract}
The giant cytoskeletal kinases of the titin-like family are emerging as key mediators of stretch-sensing in muscle. It is thought that their elastic conformational deformation during muscle function regulates both their catalysis and the recruitment of regulatory proteins to signalosomes that assemble in their vicinity. In the present article, we discuss the speciation of mechanosensory mechanisms in titin-like kinases, their scaffolding properties and the kinase/pseudokinase domain variations that define a rich functional diversity across the family.
\end{abstract}

\section{Titin-like kinases}

The cytoskeleton of muscle cells contains giant filamentous proteins of the titin-like family $(0.7-4 \mathrm{MDa})$ that mediate the sensing and transduction of mechanical signals in the myofibril. Such mechanical signals drive the development and regulation of the tissue in adaptation to physical demands. Titin-like proteins are composed of numerous Iglike domains linked in series and contain one or two kinase domains invariably located near their C-termini. Members of this family include titin and obscurin in mammals; twitchin/UNC-22, the obscurin homologue UNC-89 and the small titin TTN-1 in nematodes; twitchin in molluscs; and projectin, UNC-89 and stretchin in insects [1-3]. Despite acute variations in the length and domain organization of these proteins, their kinase regions are highly conserved, consisting of Fn (fibronectin)-linker-kinase-tail-Ig domains (Figure 1). This evolutionary conservation led researchers to anticipate a shared regulatory mechanism of the kinase domain and related signalling roles in muscle. However, recent findings point to a rich mechanistic and functional diversity among kinases of this family. In the present article, we review the currently emerging concepts regarding titinlike kinases.

\section{Intrasteric regulation by kinase-flanking extensions}

The crystal structure of the Fn-linker-kinase-tail-Ig region of Caenorbabditis elegans twitchin (TwcKR) has been elucidated recently [4]. This shows the kinase domain within its molecular context, revealing the connections that anchor

Key words: intrasteric regulation, mechanosensing, obscurin, (pseudo)kinase scaffold, titin-like kinase, twitchin.

Abbreviations used: AFM, atomic force microscopy; CPNA-1, copine domain protein atypical 1; CRD, C-terminal regulatory domain; CTD, C-terminal domain; Fn, fibronectin; MD, molecular dynamics; MURF, muscle-specific RING finger; NL, N-terminal tail; SCPL-1, small CTD phosphataselike 1; TK, titin kinase; TwcK, twitchin kinase; TwcKR, twitchin kinase region.

${ }^{1}$ To whom correspondence should be addressed (email Olga.Mayans@liv.ac.uk). and orient it in the filament: the kinase is wrapped by both $\mathrm{N}$ - and C-terminal flanking tails that pack tightly against it, with $\mathrm{Fn}$ and Ig domains tethering this kinase assembly (Figure 1b). The CRD (C-terminal regulatory domain) tail wedges itself between the two kinase lobes, blocking the binding sites for ATP and the phosphorylatable protein substrate. This CRD conformation reproduces that observed in previous structures of TwcKs (twitchin kinases) from $C$. elegans [5,6] and Aplysia [6] and human TK (titin kinase) [7]. Conversely, the TwcKR structure has revealed for the first time that the NL (N-terminal tail) is not an unstructured segment as previously assumed. Instead, it forms a 'crown' that rests on the back of the interlobular kinase hinge region, with its remaining chain folded against the $\mathrm{N}$ terminal kinase lobe, sprawling across the $\beta 1-\beta 2$ hairpin (containing the glycine-rich loop in the ATP-binding pocket) and leading into a frontal $\beta$-hairpin loop that connects the kinase assembly to the preceding Fn domain. The NL crown packs against the kinase through a conserved aromatic motif (YXXYXFXXWXXYY) (Figure 1b), but does not occupy the canonical hydrophobic pocket of AGC kinases [8]. The $\mathrm{NL}$ also establishes direct contacts with the catalytic helix $\alpha \mathrm{C}$. These multiple interactions with helix $\alpha \mathrm{C}, \beta 1-\beta 2$ hairpin and kinase hinge indicate that this extension is also a regulator of TwcK catalysis.

Kinetic data for TwcKR variants confirmed that the bare kinase domain has maximum phosphotransfer activity, whereas both NL and CRD extensions are inhibitory [4]. Separately, each segment approximately halves phosphotransfer levels, but jointly they efficiently silence catalysis. The inhibition provided by the NL segment appears to be due to a restriction of intramolecular hinge motions required for catalysis (characterized in other protein kinases $[9,10])$. In contrast, CRD-mediated inhibition is caused by the blockage of the active site (Figure 1b). It is remarkable that high levels of catalysis are observed in the presence of the CRD. However, the CRD segment that directly blocks the active site folds into a $3_{10}$ helix, a rare chain conformation that is 
Figure 1| The conserved kinase region of titin-like proteins

(a) Schematic domain composition. (b) Crystal structure of the TwcKR from C. elegons. Lateral (left) and frontal views (right; flanking Fn/Ig domains omitted to improve visibility) illustrating the packing of NL and CRD extensions against the kinase catalytic core. The interaction of the NL with the kinase hinge region (through aromatic groups), the $\beta 1-\beta 2$ hairpin (blue) containing the glycine-rich loop (G), and the helix $\alpha$ C (broken circle) is shown. The CRD- $\alpha$ R2 helix blocking the ATP-binding site is indicated by an arrowhead. Other catalytically important groups are shown and labelled as follows: catalytic aspartate (D), ATP-co-ordinating lysine (K) and DFG motif.

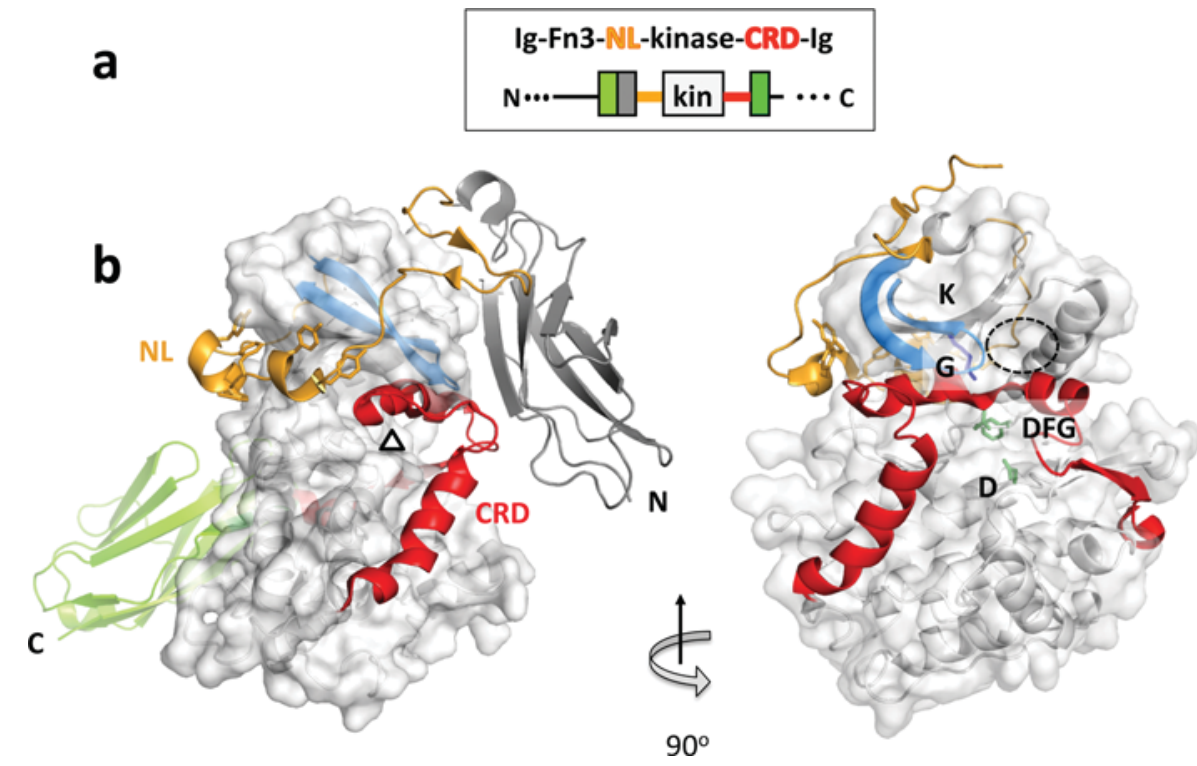

energetically unfavourable. It is conceivable that this segment might be out-competed by the substrates in the absence of the NL, possibly through a local unfolding-folding transition. In any case, activation of the NL-kinase-CRD assembly must require tail removal to some extent.

\section{Mechanoactivation}

The mechanism of tail release in titin-like kinases remains controversial. To date, protein activators that bind and displace these tails from the kinase active site have not been identified. However, it has recently been hypothesized that kinase activation is a direct result of the mechanical deformation of the cytoskeleton during myofibril stretch $[11,12]$. This hypothesis was based on AFM (atomic force microscopy) data [12-14] and force-probe MD (molecular dynamics) simulations [11] on human TK that indicated that stretch pulls the CRD from the kinase core, unfolding it and freeing the active site. It was then concluded that TK functions as a biological stress sensor, which is in agreement with the observed functional association of TK with stretch-activated pathways in the myofibril [15].

This mechanism of activation, however, does not seem to be shared by all members of the family. AFM investigations of twitchin and TTN1 (titin 1) kinases from C. elegans failed to detect the distinct unfolding of their CRD tails [16]. Steered MD simulations on TwcKR [4] then indicated that the NL in this kinase is mechanically labile, but that the
CRD is poorly responsive to stretch and acts as an integral structural component of the C-terminal kinase lobe, to which it remains bound. This, together with the observed catalytic tolerance of this tail in $\mathrm{TwcK}$, points to the possibility that the CRD might maintain extensive stabilizing contacts with the kinase domain throughout the catalytic cycle, protecting the active site against mechanical damage. In fact, stretching of the exposed kinase domain could be expected to hinder the interlobe closure motions required for catalysis, resulting in inhibition. Stretch-inhibition has been demonstrated in enzymes such as guanylate kinase, where the attachment of a DNA spring that introduced tension and halted interdomain closure also hindered catalysis [17]. In TwcK, the CRD might enable catalysis under tension by aiding intramolecular reorganization and providing fold stability.

The contrasting data on human TK and nematode TwcK correlates with actual differences in the composition, structure and interactions of their CRD tails. The CRD of TwcK is longer and more structured than that of TK and thus mechanically more resistant. This finding suggests that activation through CRD stretch-unfolding is not a generic mechanism of titin-like kinases, but that at least two mechanosensing modes exist in the family, leading to 'priming' or 'baring' of the active site. In 'priming', stretch might primarily release the NL barrier, whose removal might then permit catalysis in the presence of the CRD stabilizer. The 'baring' mechanism, on the other hand, might primarily 
Figure 2 | Scaffolding interactions of titin-like kinases

Protein interactions mediated by (a) human TK and (b) UNC-89 kinases from C. elegans. Domain colour code for TK and UNC-89 as in Figure 1. Details on interactions are given in the text. ILK, integrin-linked kinase; SRF, serum-response factor.

a

human TK

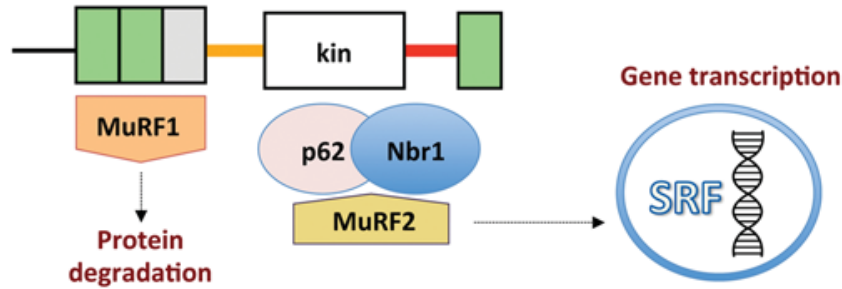

b

Extracellular signaling

$\alpha \beta$ Integrins $^{\text {Int }}$

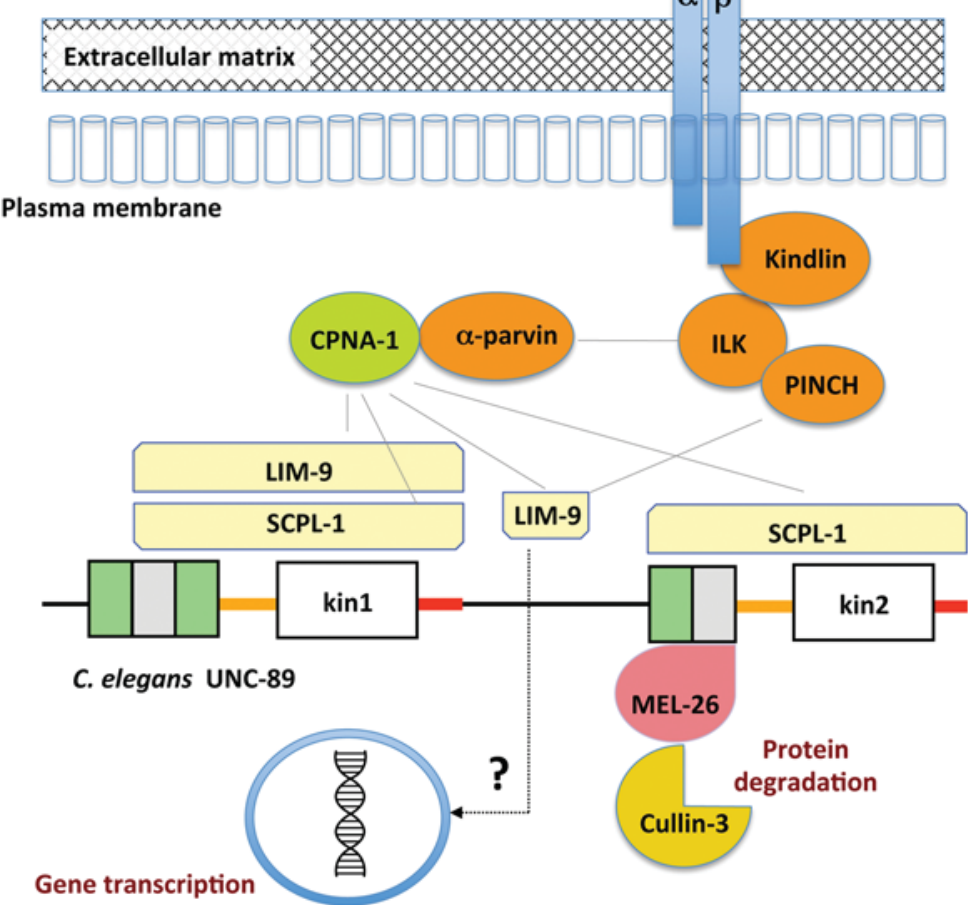

emphasize the exposure of the kinase surface, possibly also involving the release of the CRD tail as observed in TK. These two possible modes of mechanosensing might differentially affect the distinct catalytic and scaffolding roles of the kinase domains in this family.

\section{Titin-like kinases as protein scaffolds}

Recently, a number of interacting partners have been identified for titin-like kinases, indicating that scaffolding plays an important part in their signalling. Human TK interacts with the autophagosomal receptors $\mathrm{Nbr1}$ and p62, and the E3 ubiquitin ligase MuRF2 (muscle-specific RING finger 2) [15] (Figure 2a). The formation of this signalosome of turnover factors is transient and appears to be dictated by the stretch-induced conformation of TK's CRD. Upon mechanical arrest, the release of MuRF2 leads to the repression of the transcriptional response of serum- response factor, linking mechanosensing with gene expression regulation in striated muscle. In addition, the conserved IgIg-Fn tandem immediately preceding TK interacts with the ubiquitin ligase MuRF1 [18,19] that is linked to muscle atrophy $[20,21]$. Taken together, the data point to TK as an important mechanosensory signalling node in the cytoskeleton of vertebrate muscle. Notably, no binding partners are known for twitchin, TTN-1 or projectin kinases in invertebrates (the closest TK homologues) and MuRF homologues have also not been identified in that animal group. Thus, to some extent, signalling through titin-like kinases might be phylum-specific.

The kinases within the obscurin filament system (UNC89 in invertebrates) also regulate protein turnover in muscle. Obscurin/UNC-89 contain two kinase domains, PK1 and PK2, linked by a sequence of 450-1100 residues depending on the organism [22]. This interkinase linking region consistently has Ig-Fn domains N-terminal to PK2, but otherwise is 
a low-complexity sequence with high proline content and no evidence of shared homology [22]. These features are reminiscent of the well-known molecular springs of titin (PEVK, N2B and N2A [3]), suggesting that the interkinase linker is an elastic component of the obscurin/UNC-89 mechanosensory kinase node. In C. elegans UNC-89, the Ig-Fn domains preceding PK2 interact with MEL-26, a substrate-recognition protein for cullin-3 [23] (Figure 2b). Cullins are scaffolds for the assembly of the ubiquitin degradation machinery, including E3 ubiquitin ligases [24]. In early C. elegans embryos, a target of the cullin-3-MEL-26 complex is the microtubule-severing enzyme katanin (MEI1) [25]. The loss- or gain-of-function of either mel-26 or mei-1 results in disorganization of thick filaments similar to unc-89 mutants [23]. It is thought that the interaction of UNC-89 with MEL-26 inhibits the activity of the cullin3-MEL-26 complex in promoting the ubiquitin-mediated degradation of MEI-1, whose activity is required for myosin thick filament organization. Similarly, studies on an obscurinknockout mouse revealed that degradation of sAnk1.5 (important for sarcoplasmic reticulum architecture in striated muscle) is dependent upon obscurin and a cullin-3 substraterecognition protein, KCTD6 $\left(\mathrm{K}^{+}\right.$channel tetramerization domain-containing 6) [26]. These studies point to an evolutionarily related mechanism in invertebrate/vertebrate muscle by which UNC-89/obscurin regulates ubiquitinmediated protein degradation.

In addition to those interactions, each kinase domain in C. elegans UNC-89 interacts with SCPL-1 [small CTD (Cterminal domain) phosphatase-like 1], a CTD-type protein phosphatase [27] (Figure 2b). CTD phosphatases are known to regulate transcription $[28,29]$, but this finding suggested a new function for this class of proteins in muscle-specific signalling. In agreement, scpl-1 mutants had defective egglaying muscle function [27]. Furthermore, both PK1 and the interkinase region interact with LIM-9 [30], the nematode homologue of the human protein FHL (four-and-a-half LIM domains) that in vertebrate muscle associates with the elastic I-band of titin [3]. UNC-89, LIM-9 and SCPL-1 interact with CPNA-1 (copine domain protein atypical 1), a copine domain protein that localizes to integrin-adhesion sites (M-lines and dense bodies) in the body-wall muscle of C. elegans [31]. CPNA-1 binds to the M-line/dense body protein PAT-6 (actopaxin/ $\alpha$-parvin) [31], which in turn interacts with PAT-4 (integrin-linked kinase) associated with integrins [32]. Nematodes lacking CPNA-1 are embryonic lethal and display disruption of the myofilament lattice, with UNC-89 becoming misplaced into large foci [31]. Thus CPNA-1 functionally links UNC-89 kinases with integrins, thereby connecting main cytoskeletal and cellsurface sensory pathways (Figure 2b). It remains to be discovered whether this functional coupling is also present in vertebrate obscurins.

These data suggest that, through their scaffolding, titinlike kinases might act as cross-talk nodes that integrate cytoskeletal mechanics with general regulatory pathways in the myofibril. However, it must be considered that these kinases are non-diffusible, so their function must be strongly conditioned by their location in the myofibril. The importance of this observation is highlighted by the latest data on mouse obscurin kinases. It was found that a small extracellular isoform of obscurin exists at the muscle cell surface where PK2 associates with the extracellular $\beta 1$ subunit of $\mathrm{Na}^{+} / \mathrm{K}^{+}$-ATPase [33]. Although the physiological significance of this finding is unknown, it illustrates how compartmentalization might result in multiple and unsuspected functions for these kinases.

\section{Evolutionary dichotomy of kinases and pseudokinases in the titin-like family}

The catalysis of titin-like kinases remains poorly characterized. Physiological substrates are unidentified for most members of the family and several titin-like kinases are atypical, including the TK representative [34]; only twitchin and TTN-1 kinases have been shown to exhibit high levels of catalysis in vitro [4,35-37]. However, the most prominent example of catalytic divergence in this family is that of obscurin kinases that, despite high levels of sequence identity, have segregated into kinases and pseudokinases according to animal phyla. In vertebrate obscurin, PK1 and PK2 have canonical active motifs (Figure 3) and have been shown experimentally to undergo autophosphorylation [33]. In contrast, invertebrate UNC-89 kinases have atypical active sites and some might be inactive (Figure 3). UNC-89-PK1 from insects has classical ATP-binding motifs, but has lost the typical HXDXKXXN motif in the catalytic loop [38]. This has become HXGXTXXD, where the catalytic aspartate residue is replaced by an unreactive glycine residue and the frequently occurring lysine and asparagine groups assisting ATP co-ordination and phosphotransfer are replaced by threonine and aspartate. The new motif is highly conserved across all insects, pointing to the evolution of a new function or specificity of PK1 in that animal group. In contrast, in nematode UNC-89-PK1, the catalytic motif is present, but ATP-binding residues are degenerated, including the conserved lysine residue in strand- $\beta 3$ that is now a glutamine residue. This exchange might influence catalysis or specificity. Interestingly, this observation adds to the existence of $C$. elegans UNC-89 isoforms -C and -D that have truncated non-functional PK1 domains lacking the $\mathrm{N}$-terminal lobe [22]. Thus PK1 domains in insects and nematodes have potentially undergone a convergent evolution towards being catalytically altered pseudokinases, which suggests that this feature is crucial to function in invertebrate muscle. In contrast, UNC-89-PK2 has typical active motifs, apart from a somewhat modified glycine-rich loop and the fact that the catalytic aspartate residue is asparagine in several organisms (glutamate in one other member) (Figure 3 and Supplementary Figure S3 at http://www.biochemsoctrans. org/bst/041/bst0411066add.htm). Mutagenesis studies in model protein kinases revealed that such asparagine/glutamate mutations are strongly inactivating [39-41]. However, the atypical kinase JAK2 (Janus kinase 2) contains an 
Figure 3 | Catalytic motifs in kinase and pseudokinase domains of obscurin/UNC-89

The roles of the residues in these sequence motifs are described in [38]. Explicit sequence alignments are provided as Supplementary Figure S1 at http://www.biochemsoctrans.org/bst/041/bst0411066add.htm.

\begin{tabular}{|c|c|c|c|c|c|c|c|c|c|}
\hline \multirow{4}{*}{$(n=21)$} & $\begin{array}{l}\text { Human/ } \\
\text { Primate }\end{array}$ & GxGxxG & \multirow{4}{*}{ AxK } & \multirow{4}{*}{ HxDxKxxN } & \multirow{4}{*}{ DFG } & {$[Q / R] \times G \times x S$} & AxK & \multirow{4}{*}{$H \times D x K x x N$} & DLG \\
\hline & $\begin{array}{c}\text { Other } \\
\text { mammals }\end{array}$ & Gx[S/A]GxxG & & & & RxGxxS & AxK & & $\mathrm{D}[\mathrm{L} / \mathrm{F}] \mathrm{G}$ \\
\hline & Bird & GxGxxS & & & & KxGxxS & AxK & & DFG \\
\hline & Fish & GxGxx[G/S] & & & & {$[\mathrm{N} / \mathrm{A}] \times \mathrm{G} \times \mathrm{G}[\mathrm{G} / \mathrm{S}]$} & [A/L]XK & & $D[L / F] G$ \\
\hline \multirow{7}{*}{$(n=26)$} & Drosophila & \multirow{7}{*}{ GxGxxG } & AxK & HxGxTxxD & \multirow{7}{*}{ DFG } & $A x G x x S$ & VxK & $\mathrm{HxNxKxxN}$ & DFG \\
\hline & Mosquito & & AxK & HxGxTxxD & & SxGxxS & VxK & HxDxKxxN & DFG \\
\hline & Ant & & AxK & HxGxTxxD & & SxGxxS & {$[\mathrm{V} / \mathrm{I}] \mathrm{xK}$} & $\mathrm{Hx}[\mathrm{D} / \mathrm{N}] \mathrm{xKxxN}$ & DMG \\
\hline & Beetle & & AxK & HxGxTxxD & & {$[S / A] \times G \times x S$} & {$[\mathrm{~A} / \mathrm{V}] \times \mathrm{K}$} & $\mathrm{Hx}[\mathrm{D} / \mathrm{N}] \mathrm{xKx \times N}$ & D[F/M/L]G \\
\hline & Louse & & AxK & $H x[G / S] \times T \times x D$ & & $A \times G \times x S$ & $V \times K$ & $\mathrm{Hx}[\mathrm{D} / \mathrm{N}] \mathrm{xKxxN}$ & DFG \\
\hline & Butterfly & & AxK & HxGXTxxE & & WxGxxS & VxK & $\mathrm{Hx}[\mathrm{D} / \mathrm{N}] \mathrm{xKxxN}$ & DFG \\
\hline & Bee & & AxK & $\mathrm{HxG} \times \mathrm{T} \times x \mathrm{D}$ & & {$[Y / A] \times G \times x S$} & $\mathrm{VxK}$ & $\mathrm{HxDxKxxN}$ & DMG \\
\hline $\begin{array}{l}(n=4) \\
0\end{array}$ & Nematodes & ExAxxG & $L \times Q$ & $\operatorname{HxDxRxx[T/A/V]}$ & DFG & {$[F / Y / P] \times G \times x[G / S]$} & {$[A / M] \times K$} & $\mathrm{Hx}[\mathrm{D} / \mathrm{N}] \mathrm{xKxxN}$ & $D F[G / A]$ \\
\hline
\end{tabular}

asparagine residue at this location while maintaining activity, indicating that this exchange can be compensated for within the kinase machinery [42]. Hence it can be concluded that the titin-like kinase family comprises kinases, atypical kinases and barren pseudokinases with widely ranging catalytic properties. It has yet to be established which members of the family act as true mechanotransducers by mediating stretch-activated phosphotransfer and which might exploit mechanosensing primarily through other functions, e.g. conformationally regulated scaffolding.

\section{Conclusion}

Titin-like kinases form mechanosensory signalling nodes central to myofibril regulation. The speciation of their elastic regulatory segments coupled to kinase/pseudokinase catalytic variations, cellular compartmentalization and the establishment of distinct scaffolding interactions provides specificity in their sensing, supporting a range of specific muscle responses to stress stimuli.

\section{Funding}

We acknowledge the financial support of the Human Frontiers Science Programme [grant number RGP0044/2012].

\section{References}

1 Bullard, B., Linke, W.A. and Leonard, K. (2002) Varieties of elastic protein in invertebrate muscles. J. Muscle Res. Cell Motil. 23, 435-447

2 Kontrogianni-Konstantopoulos, A., Ackermann, M.A., Bowman, A.L., Yap, S.V. and Bloch, R.J. (2009) Muscle giants: molecular scaffolds in sarcomerogenesis. Physiol. Rev. 89, 1217-1267
3 Krüger, M. and Linke, W.A. (2011) The giant protein titin: a regulatory node that integrates myocyte signaling pathways. J. Biol. Chem. 286 9905-9912

4 Von Castelmur, E., Strümpfer, J., Franke, B., Bogomolovas, J., Barbieri, S. Qadota, H., Konarev, P.V., Svergun, D.I., Labeit, S., Benian, G.M. et al. (2012) Identification of an N-terminal inhibitory extension as the primary mechanosensory regulator of twitchin kinase. Proc. Natl. Acad. Sci. U.S.A. 109, 13608-13613

5 Hu, S.H., Parker, M.W., Lei, J.Y., Wilce, M.C., Benian, G.M. and Kemp, B.E. (1994) Insights into autoregulation from the crystal structure of twitchin kinase. Nature 369, 581-584

6 Kobe, B., Heierhorst, J., Feil, S.C., Parker, M.W., Benian, G.M., Weiss, K.R. and Kemp, B.E. (1996) Giant protein kinases: domain interactions and structural basis of autoregulation. EMBO J. 15, 6810-6821

7 Mayans, 0., van der Ven, P.F.M., Wilm, M., Mues, A., Young, P., Fuerst, D.O., Wilmanns, M. and Gautel, M. (1998) Structural basis for activation of the titin kinase domain during myofibrillogenesis. Nature $\mathbf{3 9 5}$, 863-869

8 Gold, M.G., Barford, D. and Komander, D. (2006) Lining the pockets of kinases and phosphatases. Curr. Opin. Struct. Biol. 16, 693-701

9 Johnson, D.A., Akamine, P., Radzio-Andzelm, E., Madhusudan, M. and Taylor, S.S. (2001) Dynamics of cAMP-dependent protein kinase. Chem. Rev. 101, 2243-2270

10 Mastersona, L.R., Masterson, L.R., Shi, L., Metcalfe, E., Gao, J., Taylor, S.S and Veglia, G. (2011) Dynamically committed, uncommitted, and quenched states encoded in protein kinase A revealed by NMR spectroscopy. Proc. Natl. Acad. Sci. U.S.A. 108, 6969-6974

11 Gräter, F., Shen, J., Jiang, H., Gautel, M. and Grubmüller, H. (2005) Mechanically induced titin kinase activation studied by force-probe molecular dynamics simulations. Biophys. J. 88, 790-804

12 Puchner, E.M., Alexandrovich, A., Kho, A.L., Hensen, U., Schäfer, L.V., Brandmeier, B., Gräter, F., Grubmüller, H., Gaub, H.E. and Gautel, M. (2008) Mechanoenzymatics of titin kinase. Proc. Natl. Acad. Sci. U.S.A. 105, 13385-13390

13 Puchner, E.M. and Gaub, H.E. (2010) Exploring the conformation-regulated function of titin kinase by mechanical pump and probe experiments with single molecules. Angew. Chem. Int. Ed. 49, 1147-1150

14 Stahl, S.W., Puchner, E.M., Alexandrovich, A., Gautel, M. and Gaub, H.E. (2011) A conditional gating mechanism assures the integrity of the molecular force-sensor titin kinase. Biophys. J. 101, 1978-1986 
15 Lange, S., Xiang, F., Yakovenko, A., Vihola, A., Hackman, P., Rostkova, E., Kristensen, J., Brandmeier, B., Franzen, G., Hedberg, B. et al. (2005) The kinase domain of titin controls muscle gene expression and protein turnover. Science 308, 1599-1603

16 Greene, D.N., Garcia, T., Sutton, R.B., Gernert, K.M. and Benian, G.M. (2008) Single-molecule force spectroscopy reveals a stepwise unfolding of Caenorhabditis elegans giant protein kinase domains. Biophys. J. 95, 1360-1370

17 Choi, B., Zocchi, G., Wu, Y., Chan, S. and Perry, L.J. (2005) Allosteric control through mechanical tension. Phys. Rev. Lett. 95, 078102

18 Centner, T., Yano, J., Kimura, E., McElhinny, A.S., Pelin, K., Witt, C.C., Bang, M.L., Trombitas, K., Granzier, H., Gregorio, C.C. et al. (2001) Identification of muscle specific ring finger proteins as potential regulators of the titin kinase domain. J. Mol. Biol. 306, 717-726

19 Mrosek, M., Labeit, D., Witt, S., Heerklotz, H., von Castelmur, E., Labeit, S. and Mayans, 0. (2007) Molecular determinants for the recruitment of the ubiquitin-ligase MURF-1 to M-line titin. FASEB J. 21, 1383-1392

20 Bodine, S.C., Latres, E., Baumhueter, S., Lai, V.K., Nunez, L., Clarke, B.A., Poueymirou, W.T., Panaro, F.J., Na, E., Dharmarajan, K. et al. (2001) Identification of ubiquitin ligases required for skeletal muscle atrophy. Science 294, 1704-1708

21 Mayans, 0. and Labeit, S. (2012) MuRFs: specialized members of the TRIM/RBCC family with roles in the regulation of the trophic state of muscle and its metabolism. Adv. Exp. Med. Biol. 770, 119-129

22 Small, T.M., Gernert, K.M., Flaherty, D.B., Mercer, K.B., Borodovsky, M and Benian, G.M. (2004) Three new isoforms of Caenorhabditis elegans UNC-89 containing MLCK-like protein kinase domains. J. Mol. Biol. 342, 91-108

23 Wilson, K.J., Qadota, H., Mains, P.E. and Benian, G.M. (2012) UNC-89 (obscurin) binds to MEL-26, a BTB-domain protein, and affects the function of MEl-1 (katanin) in striated muscle of Coenorhabditis elegons. Mol. Biol. Cell 23, 2623-2634

24 Bosu, D.R. and Kipreos, E.T. (2008) Cullin-RING ubiquitin ligases: global regulation and activation cycles. Cell Div. 3, 7

25 Pintard, L., Willis, J.H., Willems, A., Johnson, J.L., Srayko, M., Kurz, T., Glaser, S., Mains, P.E., Tyers, M., Bowerman, B. and Peter, M. (2003) The BTB protein MEL-26 is a substrate-specific adaptor of the CUL-3 ubiquitin-ligase. Nature 425, 311-316

26 Lange, S., Perera, S., The, P. and Chen, J. (2012) Obscurin and KCTD6 regulate cullin-dependent small ankyrin-1 (sAnk1.5) protein turnover Mol. Biol. Cell 23, 2490-2504

27 Qadota, H., McGaha, L.A., Mercer, K.B., Stark, T.J., Ferrara, T.M. and Benian, G.M. (2008) A novel protein phosphatase is a binding partner for the protein kinase domains of UNC-89 (obscurin) in Coenorhobditis elegons. Mol. Biol. Cell 19, 2424-2432

28 Lin, P.S., Marshall, N.F. and Dahmus, M.E. (2002) CTD phosphatase: role in RNA polymerase II cycling and the regulation of transcript elongation. Prog. Nucleic Acid Res. Mol. Biol. 72, 333-365

29 Meinhart, A., Kamenski, T., Hoeppner, S., Baumli, S. and Cramer, P. (2005) A structural perspective of CTD function. Genes Dev. 19, 1401-1415
30 Xiong, G., Qadota, H., Mercer, K.B., McGaha, L.A., Oberhauser, A.F. and Benian, G.M. (2009) A LIM-9 (FHL)/SCPL-1 (SCP) complex interacts with the $\mathrm{C}$-terminal protein kinase regions of UNC-89 (obscurin) in Coenorhabditis elegans muscle. J. Mol. Biol. 386, 976-988

31 Warner, A., Xiong, G., Oadota, H., Rogalski, T., Vogl, A.W., Moerman, D.G. and Benian, G.M. (2013) CPNA-1, a copine domain protein, is located at integrin adhesion sites and is required for myofilament stability in Coenorhabditis elegans. Mol. Biol. Cell 24, 601-616

32 Lin, X., Qadota, H., Moerman, D.G. and Williams, B.D. (2003) C. elegans PAT-6/actopaxin plays a critical role in the assembly of integrin adhesion complexes in vivo. Curr. Biol. 13, 922-932

$33 \mathrm{Hu}$, L.Y. and Kontrogianni-Konstantopoulos, A. (2013) The kinase domains of obscurin interact with intercellular adhesion proteins. FASEB J. 27, 2001-2012

34 Scheeff, E.D., Eswaran, J., Bunkoczi, G., Knapp, S. and Manning, G. (2009) Structure of the pseudokinase VRK3 reveals a degraded catalytic site, a highly conserved kinase fold, and a putative regulatory binding site. Structure 17, 128-138

35 Lei, J., Tang, X., Chambers, T.C., Pohl, J. and Benian, G.M. (1994) Protein kinase domain of twitchin has protein kinase activity and an autoinhibitory region. J. Biol. Chem. 269, 21078-21085

36 Flaherty, D.B., Gernert, K.M., Shmeleva, N., Tang, X., Mercer, K.B., Borodovsky, M. and Benian, G.M. (2002) Titins in C. elegans with unusual features: coiled-coil domains, novel regulation of kinase activity and two new possible elastic regions. J. Mol. Biol. 323, 533-549

37 Heierhorst, J., Tang, X., Lei, J., Probst, W.C., Weiss, K.R., Kemp, B.E. and Benian, G.M. (1996) Substrate specificity and inhibitor sensitivity of $\mathrm{Ca}^{2+} /$ S100-dependent twitchin kinases. Eur. J. Biochem. 242 454-459

38 Endicott, J.A., Noble, M.E. and Johnson, L.N. (2012) The structural basis for control of eukaryotic protein kinases. Annu. Rev. Biochem. $8 \mathbf{1}$ 587-613

39 Skamnaki, V.T., Owen, D.J., Noble, M.E., Lowe, D.E., Lowe, G., Oikonomakos, N.G. and Johnson, L.N. (1999) Catalytic mechanism of phosphorylase kinase probed by mutational studies. Biochemistry $\mathbf{3 8}$ 14718-14730

40 Cole, P.A., Grace, M.R., Phillips, R.S., Burn, P. and Walsh, C.T. (1995) The role of the catalytic base in the protein tyrosine kinase Csk. J. Biol. Chem. 270, 22105-22108

41 Ablooglu, A.J., Frankel, M., Rusinova, E., Ross, J.B.A. and Kohanski, R.A. (2001) Multiple activation loop conformations and their regulatory properties in the insulin receptor's kinase domain. J. Biol. Chem. 276 46933-46940

42 Bandaranayake, R.M., Ungureanu, D., Shan, Y., Shaw, D.E., Silvennoinen, O. and Hubbard, S.R. (2012) Crystal structures of the JAK2 pseudokinase domain and the pathogenic mutant V617F. Nat. Struct. Mol. Biol. 19, 754-759 